\title{
VALUE ANALYSIS USING FUNCTION ANALYSIS SYSTEM TECHNIQUE(FAST)
}

\author{
Aditya Pokhriyal \\ Third-year Student, Mechanical and Automation Engineering, \\ Dr. Akhilesh Das Gupta Institute of Technology and Management, New Delhi, India
}

\begin{abstract}
Value Engineering is a systematic process to improve the value of the product. It is the functional concept which distinguishes Value Engineering from many approaches to cost reduction. Function analysis is part of value analysis as its purpose is to identify product functions compared to cost and price; design and construction assessment is done. FAST is a Technique that identifies and illustrates the essential function to accommodate a design purpose. This paper will provide instruction to build FAST diagrams for the enhancement of the project.
\end{abstract}

Keywords - Value, Function, critical path, High order, and low order

\section{INTRODUCTION}

Designing a product with high value at a minimum cost has become a major demand for the industry for years. To solve this problem Value Engineers used an advance technique called FAST (Function Analysis System Technique) in which graphical representation is created to simplify the functions of the component and structures a critical path which constitutes of all primary function.

Value

The value of a function is defined as the relationship of cost to performance. Frederick S. Sherwin et al.(1968)in their work emphasize on the value of the object.

$$
\text { Max Value }=\frac{\text { Max Performance }}{\text { Min Cost }}
$$

Function

A Function can be defined as the result or action desired by the customer. It constitutes of two fundamentals: noun and verb. Noun defines the object present in the assembly of the product whereas, on the other hand, The Verb defines the action done by the object.

\section{FUNCTION $=$ VERB + NOUN \\ Example:- A lamp post on \\ the street Illuminating:- verb \\ Light:- noun}

\section{INTRODUCTION TO FAST}

In 1964, Charles W.Bytheway developed FAST Diagrams by building a flow chart which was interlinked by the functions of the component in high to low order, verb-noun functions to describe complex systems.

The main applications of the FAST diagram are to help in checking for and identify missing function value Engineering, moreover, it simplifies the list of functions generated by the object, moreover, it allows a large number of dissimilar technical backgrounds' professionals to effectively communicate and resolve issues.

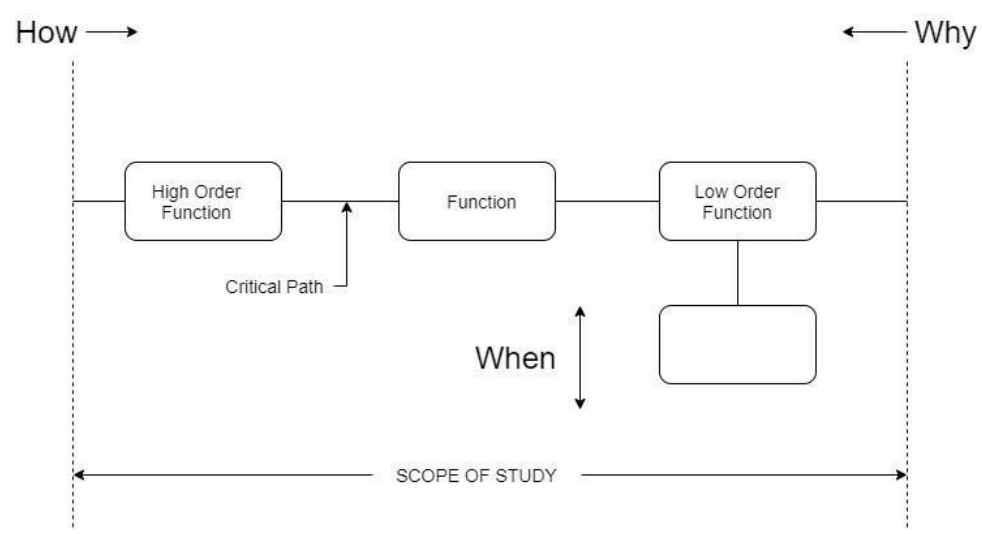

Fig:01 Basic layout for FAST diagram

\section{PROCEDURE FOR DESIGNING A FAST DIAGRAM}

The steps necessary to construct the FAST diagram are as follows:

Enlist the functions of the assembly (using noun and verb). Determine the position of the function by next higher and lower function cards by specifically answering the following logic questions:

"How?": How is function performed?

"Why?": Why is function performed?

"When?": When is function attained?

By this method, we can check the proper order of the functions in the diagram from right to left and also determine the completeness of the diagram by assuring that all functions are accounted and applied to each function and the FAST diagram continued until we arrive at a function that is an accepted interface function for the scope of the problem.

Function Determination:

This system provides us with the determination logic to determine the basic function and the higher- and lower-level functions, as well as the supporting systems. The use of this system requires the construction of a FAST diagram utilizing 


\section{International Journal of Engineering Applied Sciences and Technology, 2020 Vol. 5, Issue 1, ISSN No. 2455-2143, Pages 664-665 \\ Published Online May 2020 in IJEAST (http://www.ijeast.com)}

the determination logic questions "How?", "Why?", and "When?"

\section{Critical Path Determination}

When all the determination logic questions "How?" and "Why?" are answered for each function, we have arranged all the functions at higher and lower levels that are required to perform the highest-level basic function from left to right of the diagram. The critical path identifies the functions that are the result of other functions to be performed.

\section{Supporting Function Determination}

After the critical path functions have been selected and positioned on the FAST diagram, the remaining functions from the list in earlier step are placed vertically on the diagram by implementing the question "When?".

Exclude alternatives and choices, not in the existing system.

The selected method to perform a function might, brings other functions into existence.

\section{For Example:}

The function analysis system technique for sending a feature phone, A feature phone is an electronic device that is used to communicate with each other using wireless connections. At first, all the functions of the stapler are examined as follows:

- Charging the battery of the phone

- $\quad$ Ailing the receiver's phone number

- Sending messages

- Transmission of a message using a wireless medium

- Message received by the receiver.

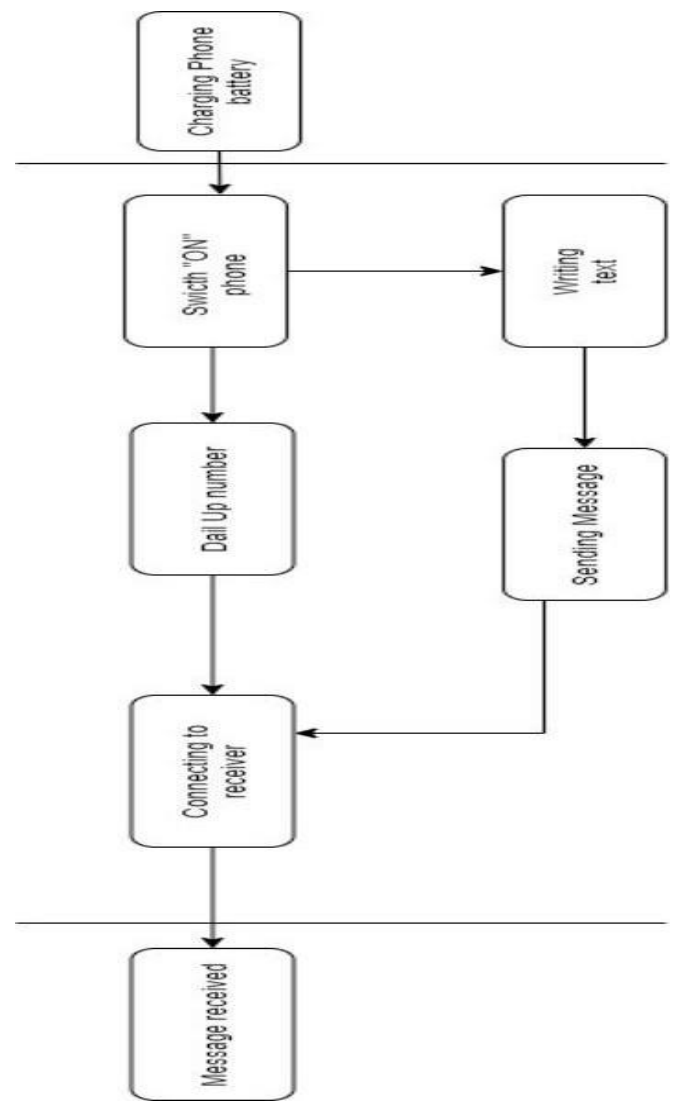

\section{CONCLUSION}

The function analysis system technique is useful in determining the function interrelation in analyzing an entire system or a major portion of a system and gives an understanding of the interaction of function and cost. The value engineering team can draw existing systems or existing designs, as well as system and purpose design. Value Engineering is best carried out when the proposed design can be defined fully, just prior to the phase where final production drawings are in process.

\section{ACKNOWLEDGMENT}

I wish to acknowledge the contributions of everyone, especially authors and publishers, whose works were consulted during the execution of this study and others who contributed directly or indirectly to the success of this work. I would also like to show my gratitude toward Dr. Inderdeep Singh, Department of Mechanical and Industrial Engineering IIT Roorkee, India.

\section{REFERENCE}

1. Lawrence D. Miles, 1993Techniques of Value Analysis and Engineering,pg-299.

2. L.D.Miles,1962,Techniques of Value analysis and Engineering,pg-02

3. Anil Kumar Mukhopadhyaya ,2009Value Engineering Mastermind From Concept to Value Engineering Certification FAST diagrams,pg-105-108

4. John Borza,2011, The Foundation for Creating Effective Function Models, trizondeteroit2011

5. Mohamed Abdelghany,Racha Rahwan,Ibrahim Abotaleb,AmrFathy,Ahmed AlBughd adi,2018, Value Engineering Applications to Improve Value in Residential Projects,pg-900

6. Frederick S. Sherwin,1968, The Value Engineering Functional Approach Techniques,pg-43

7. Value Engineering, its Contribution to Profitability by Rex Ferguson, C.Eng., M.I.Mech.E., Mem.A.S.M.E.

8. Lee, M.-J., Lim, J.-K., \& Hunter, G. ,2010, Performancebased value engineering application

9. to public highway construction. KSCE Journal of Civil Engineering, , 261-271.

10. Charles W. Bytheway ,2007,FAST Creativity Innovation: Rapidly Improving Processes, Product Development and Solving Complex Problems pg-12.

11. Perdana Miraj1, Ahmad Dofir1, Azaria Andreas1, Mohammed Ali Berawi2, Saipol Bari BinAbd Karim 2019 SYSTEMATIC REVIEW OF VALUE ENGINEERING RESEARCH STUDIES ININDONESIA,Academia.

12. Park C.-S., Kim, H.-J., Park, H.-T., Goh, J.-H., \& Pedro, A. (2017). BIM-based idea bank for managing value engineering ideas, 35 .

13. Deepak Dhounchak,Lalit Kumar Biban,2017,A REVIEW ARTICLE ON VALUE ENGINEERING,jet 